\title{
Knowledge-Based Lifecycle Management Approach for Product Service Systems (PSS)
}

\author{
Michael Abramovici, Youssef Aidi, and Hoang Bao Dang \\ Chair of IT in Mechanical Engineering, Ruhr-University Bochum, Germany \\ \{Michael.Abramovici, Youssef.Aidi, Bao.Dang\} @itm.rub.de
}

\begin{abstract}
Product Service Systems (PSS) are new industrial offerings which integrate a product and related services into a customer-specific bundle and offer the customer an overall solution to a specific issue. The lifecycle of PSS is characterized by merged product and service structures and a close interaction among the provider, the suppliers, and the customer, not only in the development phase but especially during the operation of PSS. Therefore a lifecycle management approach for PSS has to merge both the virtual and real (operational) lifecycle of PSS. To reach that goal operational knowledge about a PSS instance must be gathered, aggregated, managed, and used by enhanced lifecycle management methods in order to support providers, suppliers, and customers by developing, delivering and operating PSS. The paper in hand presents a knowledge-based lifecycle management approach for PSS, which considers an ontological representation of PSS knowledge and 3 knowledgebased lifecycle methods to make engineering processes adaptive, to provide the involved actors with the appropriate PSS knowledge and support the stakeholders in their decision making processes. The components of this approach have been identified as key lifecycle management methods for managing PSS. They are still being developed within the collaborative research center TR29 funded by the German Research Foundation (DFG), who investigate the overall engineering of PSS.
\end{abstract}

\section{Introduction}

Due to emerging global markets, companies must develop suitable products that meet the regional requirements of various customers in each country, and face the rapid technological development, unpredictable social and political changes, as well as compliance. The reaction of the industry to these discerning demands is new overall offerings called Product Service Systems (PSS). PSS are defined as "an integrated industrial product and service offering that delivers value in use" [1]. At the core of this approach is the increase in provider and customer benefits through the integration of products and services. This definition puts emphasis on value in use. Instead of spending money on the product as a mere physical artifact, the customer nowadays wants to pay for the use of the product and its resulting value to reduce inherent risks. Compared to traditional products, and driven by growing customer pressure due to newly emerging availability and result-oriented business models, the PSS provider is 
responsible both for developing and delivering PSS [3]. In Contrast to common PLM solutions PLM approach for managing products a PSS lifecycle Management has to deal with merged product and service structures and focus more on the operation phase where a close interaction among the provider, service suppliers, and customers is required. Within the Collaborative Research Center TR29 funded by the German Research Foundation (DFG), a lifecycle management approach has been developed which is presented in this paper. Based on own surveys carried out [2][4], three key knowledge-intensive processes have been identified that constitute the core PSS lifecycle methods: Adaptive change processes, use information feedback processes and decision processes. The realization of those processes is based first on an ontological approach for the integration and management of PSS information and on an open service-oriented architecture for a loose integration in the existing IT environment.

\section{Requirement to a PSS Lifecycle Management and Challenges}

Over the last decade, Product Lifecycle Management (PLM) has become the central management approach of engineering processes and data, and it has been used as a company-wide integration platform. Current PLM solutions are focused mainly on the support of administrative information flow processes (e.g. release and change management) within the development phase. The solutions offer data models and methods which are used for the lifecycle management of product classes or families of similar products and reflect the provider's perspective on the lifecycle. A variety of research works are currently ongoing to extend the PLM approach and cover after sales phases like the closed loop PLM approach [13] but without taking into account the management of service components. The development and management of services, however, have only been considered by few methods like Service Blue Printing [5] or Service CAD [6]. An overall lifecycle management approach that supports the integrated engineering of products and services and considers the properties of PSS engineering is still required.

Depending on the PSS business model the PSS provider also becomes responsible for the operation and later phases of the PSS lifecycle, which increases both revenue and risks. In order to keep up the performance of the PSS offering, single PSS instances are changing continuously during the delivery phase [7]. Hence, providers must be able to adapt their PSS to unpredictable boundary condition changes swiftly, and therefore require adaptive change management that does not define change processes a priori. In order to make the appropriate improvements by reducing risks, the involved actors must carry out different analysis activities. Hence, they must be informed in detail about the real lifecycle of the concerned PSS instance. Thus a feedback flow of PSS use information must be established, which merges information of the real PSS lifecycle from the operation phase with information of the virtual PSS lifecycle in the development phase. Stakeholders on tactical and strategic enterprise levels are confronted with high risk decisions (e.g. the definition of the PSS business model). They need a powerful decision support system that gathers different technical and financial information from different sources and aggregates it for comprehensive analysis. 


\section{Overall PSS Lifecycle Management Approach}

Based on the requirements defined and own surveys, an overall PSS lifecycle management approach has been developed within the collaborative research center TR29 funded by the German Research Foundation (DFG). This approach is based on the established PLM approach and introduces the knowledge component as a key factor to offer the required support for managing PSS engineering. It proposes three knowledge-based assistants which address the requirements mentioned above and an ontological information modeling approach (cf. Fig. 1).

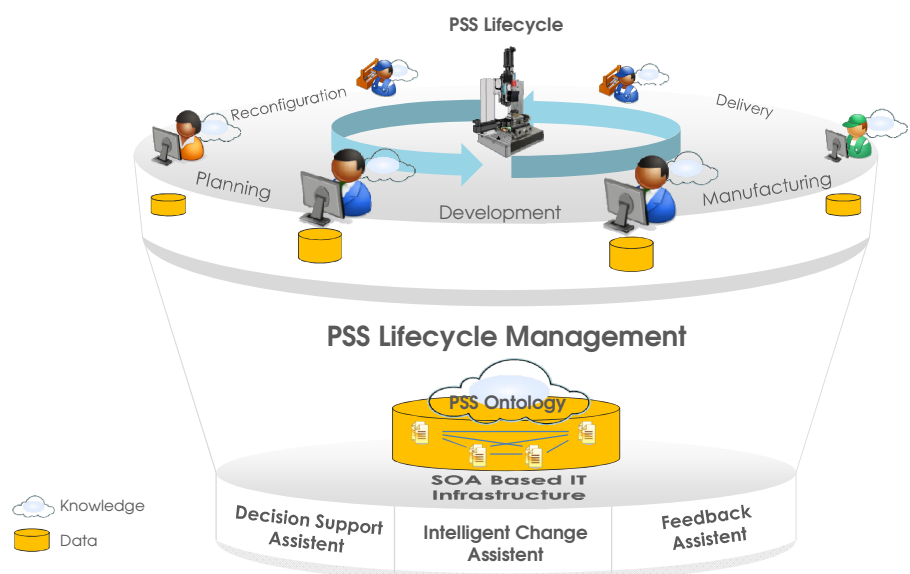

Fig. 1. Concept for the knowledge-based, overall PSS Lifecycle Management

The intelligent Change Assistant which responds to the non-deterministic properties of PSS change processes. The use information feedback assistant has been introduced, which generates and leads use knowledge from the operating phase back into the development phase, for continuous improvement of the offered PSS. And a decision support assistant supporting tactical and strategic decision makers. Furthermore, an ontological approach has been introduced to map specific PSS ontologies onto existing ontologies. To reduce the complexity of data and system integration, the proposed approach follows an SOA paradigm.

In the following sections, the components of the PSS lifecycle management approach are investigated.

\subsection{Intelligent Change Assistant}

A PSS provider must be able to adapt PSS offerings to quickly respond to unforeseeable changes in the environment throughout the lifecycle, which are caused by various drivers [8]. Hence, a prompt reaction to these unpredictable changes along the overall lifecycle of PSS has a significant impact on the economic success of a company and her network partners [9]. Each PSS change process can be unique. Different technical, organizational, and financial circumstances, the relationships and mutual influence of 
product and service components [8] in particular, influence how the change goal can be reach. In contrast to existing deterministic and firmly planned, current Engineering Change Management processes, the PSS Change Management aim is a goal-oriented real-time definition of executable change process activities and their execution priorities depending on change contents, context, objectives, and current conditions [2] (e.g. continuous real-time plan-and-execute rather than static plan-and-execute). This approach allows a prompt configuration and immediate start-up [10] while taking into consideration the great uncertainties that arise in the development and delivery phases during the execution of PSS change processes. An example of an adaptive PSS Change Assistant of that kind has been developed based on a new goal-oriented process management approach defined by Daimler AG and Whitestein Technologies [11].

- First and foremost, the processes are to capture and characterize the defined business goal, independent of the solution. Goals can be split into further sub goals.

- Each goal is assigned a generic implementation plan, which merely consists of independent tasks or activities without any predefined execution sequence or priorities.

- The specifications of tasks and activities, and the order in which they are carried out are determined during process execution, in real time, and depending on the main process issues and the current situation (rules) of the process.

Within the process, the tasks or activities are defined as intelligent agents. They represent the right road to the (sub) goal, appropriately, independently, and subject to the rules [12] (cf. Fig. 2). These rules are represented in the PSS Change Ontology, which describes the change activities and their interaction under different circumstances. Through reasoning of the PSS Change Ontology the PSS Change Assistant defines possible activity chains and permanently checks the achievement of the phase goals. In case of trouble alternative activities can be found and the change process can be adjusted to the new circumstances.
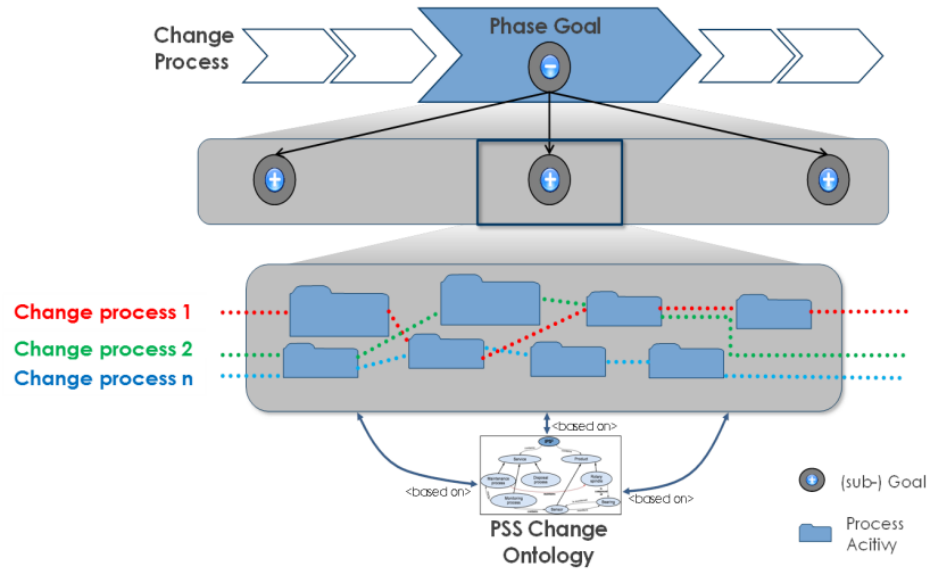

Fig. 2. Goal-oriented process modeling 


\subsection{PSS Feedback Assistant}

To comply with the PSS contract, the PSS provider has to provide any required service to ensure the delivery of the promised value of the product use. Furthermore continuous and effective improvements can enhance the PSS performance, which increases both customer and provider proceeds. The availability of product use data creates new opportunities by generating product use knowledge as a feedback for PSS developers and PSS operation planners. The offering of PSS enables the PSS provider to have access to and control over any product use and service process data. Technical progress in embedded micro-sensors facilitates a continuous systematic acquisition of product use data for the control and planning of the machine operation and maintenance processes [13] [14]. At present, the improved design of new product generations only uses isolated and unsystematic feedback from customers, retailers, or service partners, which mainly refers to warranty cases, complaints, or product recalls [15]. As product use information is not exploited systematically, new product generations are still suboptimal or over-engineered [14] [16]. The PSS Feedback Assistant (cf. Fig. 3) considers the exploitation of a large amount of individual, similar PSS components data to aggregate using Extract, Transformation and Loading (ETL) methods [14]. This is a necessary step to provide crucial knowledge to the product developer later on [17]. The assistant considers the following design-relevant information:

- product instance use information, like use incidents (i.e. failures, breakdowns, cracks, leaks), operational parameters (i.e. operation duration and cycles, rotation speed, temperature, vibrations), or resource consumption (i.e. energy, materials)

- product instance workspace information, like parent assembly or neighbor influencing parts

- product service data (i.e. repair, maintenance, overhaul events, replacement of parts)

- product user/operator data (i.e. personnel data, qualifications, workload) [15]

Based on the central database, the following methods have been realized [14]:

- Analysis methods provide statistical data analysis methods, e.g. for overlooking key indicators, generating use profiles and their visualizations.

- Diagnostic methods provide knowledge-based methods (Bayesian Networks) to detect causes of failure. A Bayesian Network is a probabilistic graphical model that represents a set of variables and their probabilistic dependencies. [18].

These methods support developers within a PSS change process in choosing the appropriate PSS configuration alternative. The modularity of PSS leads to more than one possible solution, and various criteria must be met in decision-making, which alternatively guarantee the desired performance under real boundary conditions. 


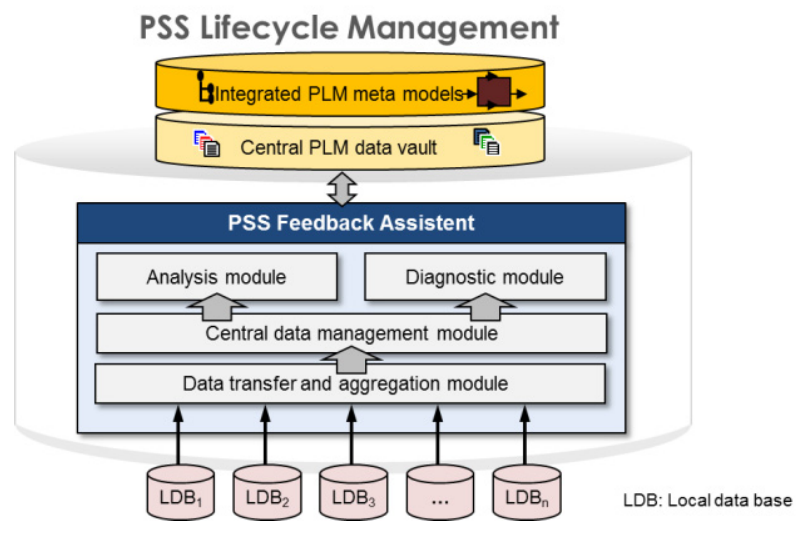

Fig. 3. Concept for the PSS Feedback Assistant

\subsection{PSS Executive Decision Assistant}

The increasing interdisciplinary of modern products and the introduction of product service offerings (PSS), as well as the related close interactions among providers, suppliers, and customers, pose new challenges to managers and decision makers [19]. On one average day, decision makers like managers are involved in a variety of tasks such as meetings, appointments, business negotiations, and report-reading [20]. Their experience cannot be fully used to manage such interdisciplinary and highly complex decision processes. First, the most technical aspects of products and characteristics of engineering processes influence the economic end result. On the other hand, the required information within the decision processes is distributed on many systems, and there is no transparency about its origin. Furthermore, current commercial systems like Business Intelligence solutions mostly focus on financial information and business operations, and thus cannot fully meet the requirements of decision makers in modern companies [21]. The employed applications (such as ERP, SCM, CRM, PLM, etc.) at tactical and strategic levels contain a high amount of valuable data and information for decision making at the respective level. However, only few provide specific modules to meet the information demand of decision makers and managers. These modules can also analyze the data stored in the single system.

The PSS Executive Decision Assistant supports decision makers on tactical and strategic level through gathering any required data within their decision processes from different data sources at provider, customer, and supplier level. That data is analyzed and aggregated to provide a holistic and transparent PSS Key Performance Indicator [22] (cf. Fig. 4). 


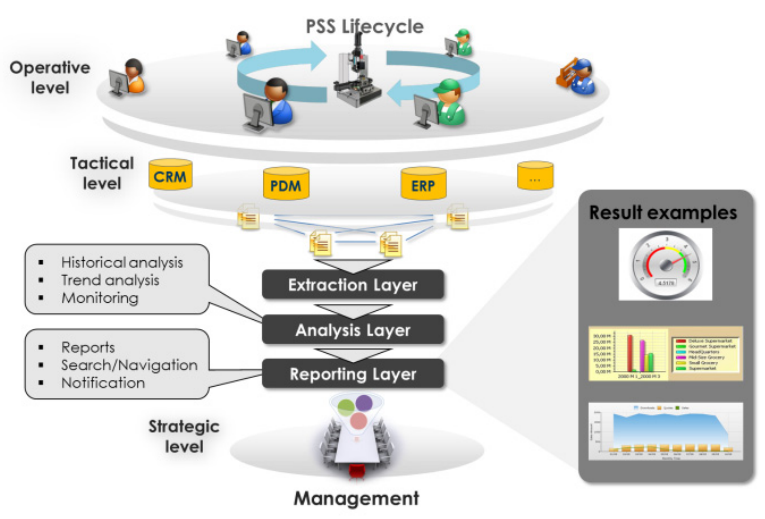

Fig. 4. Concept for the PSS Executive Decision Support Assistent

The following functions are required:

- Extraction to gather data from different internal and external sources involved in the whole lifecycle such as ERP, SCM, etc.

- Analysis by means of mathematical methods and based on existing internal and external data to make trend analyses and forecasts, which are used as valuable references for future decisions.

- Reporting to automatically provide decision makers with the right reports at the right time, according to their issues at the appropriate decision level and respective situation. That way, unnecessary reports are avoided and the time to prepare the reports is greatly shortened.

\subsection{The Ontological Approach of PSS Lifecycle Management}

One of the most important goals of the PLM concept and the PSS-LM concept is the integration of different domain-specific systems. Throughout the lifecycle of PSS, different partners are involved. Some of them may be part of the PSS network right from the start while others join in at later points of the PSS use. This poses the challenge to supply all partners with relevant information and to integrate their systems accordingly, so that information can be gathered and exchanged efficiently. As the partner's systems and IT infrastructure are diverse and may change during PSS use, a system-independent approach is required.

To integrate information from different sources of this dynamic PSS network and to support the above-mentioned intelligent assistants, an 'over-the-top' integration concept (cf. Fig. 5) has been developed that extends the semantic aspect of normal relational PLM databases. This concept enables coping with the challenges of flexible data model extensibility and management of implicit knowledge. Furthermore, this knowledge-based approach includes a PSS ontology that integrates PSS-relevant data 


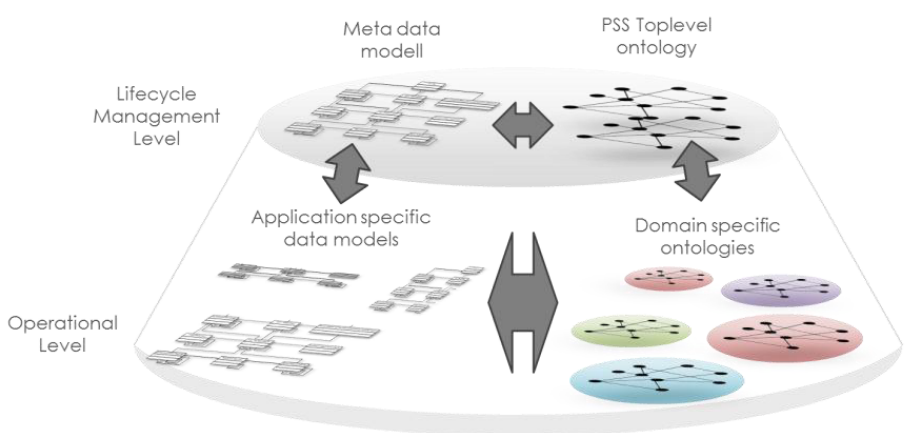

Fig. 5: 'Over-the-top' integration concept

via predefined rules and axioms. There are two relevant layers in the PSS ontology: The first layer describes eleven important PSS concepts and the relationships among them (i.e. product, service, product-service module, PSS, requirement, business models, resource, needs, functions, support information, and PSS-specific knowledge). This is called a 'PSS top-level ontology'. A comprehensive description of the eleven PSS ontology concepts can be found in a previous publication [23]. The web ontology language OWL 2 DL has been used to describe the PSS top domain ontology, as it is easily extensible and backward compatible. The description of the relations serves as a basis for the integration of domain-specific ontologies and systems from operative levels. In order to connect to other ontologies or to extend the PSS ontology, a mapping method has been developed. This method considers the four key components of the ontology concept (class including subclasses, relation including axioms, instances, and properties) as well as the influential role of the knowledge engineer. The relationships between the four key components are analyzed to determine whether a class of ontology 1 can be mapped onto another class, instance, or properties of ontology 2 , and thus determine the required relations or axioms. In order to archive and maintain the concepts/classes and relationships among them, graph database technology is used as it offers the ability to work efficiently with relationships. Moreover, the mapping method integrates data from heterogeneous sources in order to discover implicit knowledge. The knowledge engineer can manipulate the results of the mapping process. The second layer contains other ontologies, which are of importance in order to execute intelligent PLM-extended methods (e.g. adaptive change management, SOA Service Management with Function-As-Service Ontology - FAS Ontology). By means of the PSS ontology, new semantic information is provided that is based on information stemming from domain-specific data sources and generated by predefined rules and axioms.

\section{Conclusion}

The overall PSS Lifecycle Management is the central approach for the integration of PSS engineering data, processes, IT tools, and actors involved in the entire lifecycle, 
both on the provider's and the customer's sides. The outlined approach takes into account the main development stream driven by different economic and technological drivers. The flexible SOA-based IT infrastructure and the ontological approach for PSS knowledge exploration provide a suitable platform for the described knowledgebased assistants. That way, PSS use information can be lead back to earlier engineering phases. In order to make change processes more efficient, the adaptive PSS Change Assistant provides a real-time generation of change workflows considering current constraints. In addition, decision makers on tactical and strategic levels are supported by PSS Executive Decision Support which collects enterprise data from different sources and aggregates it for a high transparency of PSS lifecycle processes.

\section{References}

[1] Datta, P.P., Roy, R.: Cost Modelling Techniques for Availability Type Service Support Contracts: A Literature Review and Empirical Study. In: Proceedings of the 1st CIRP IPS $^{2}$ Conference, Cranfield, UK, pp. 216-223 (2009)

[2] Abramovici, M., Aidi, Y.: Next Generation Product Lifecycle Management (PLM). In: Proceedings of the 5th International Conference on Integrated Systems Design and Technology, Mallorca, Spain (2012)

[3] Mont, O.: Clarifying the concept of product-service systems. Journal of Cleaner Production 10(3), 237-245 (2002)

[4] Abramovici, M., Schulte, S.: Study "Benefit of PLM - The Potential Benefits of Product Lifecycle Management in the Automotive Industry". ITM Ruhr-University Bochum, IBM BSC, Detroit (2007)

[5] Arai, T., Shimomura, Y.: Service CAD System-Evaluation and Quantification. CIRP Annals - Manufacturing Technology 1, 463-466 (2005)

[6] Shostack, G.L.: Service Design in the Operating Environment. In: Developing New Services, Chicago (1984)

[7] Främling, K., Chong, B.B., Brusey, J.: Globally unique product identifiers - requirements and solutions to product lifecycle management. In: Proceedings of the 12th IFAC Symposium on Information Control Problems in Manufacturing (INCOM), Saint-Etienne, France, pp. 47-51 (2006)

[8] Abramovici, M., Bellalouna, F., Goebel, J.C.: Adaptive change management for industrial product service systems $\left(\mathrm{IPS}^{2}\right.$ ). In: Proceedings of the TMCE 2010, Ancona, Italy (2010)

[9] Brown, J., Boucher, M.: Engineering Change Management 2.0: Better Business Decisions from Intelligent Change Management (2007)

[10] Kernland, M., Hoeffleur, O., Felber, M.: The Agility Challenge in Business Process Management. Product Data Journal 1/2008, 40-42 (2008)

[11] Burmeister, B., Arnold, M., Copaciu, F., Rimmassa, G.: BDI-Agents for Agile GoalOriented Business Processes. In: Proceedings of the 7th International Conference on Autonomous Agents and Multiagent Systems (AAMAS), Estorial, Portugal (2008)

[12] Roa, A.S., Geogreff, M.P.: BDI Agents: From Theory to Practice. In: Proceedings of the 1st International Conference on Multi-Agent Systems, San Francisco, USA (1995)

[13] Dimitris, K.: Closed-loop PLM for intelligent products in the era of the Internet of things. Computer-Aided Design 43, 479-501 (2011) 
[14] Abramovici, M., Lindner, A., Walde, F., Fathi, M., Dienst, S.: Decision support for improving the design of hydraulic systems by leading feedback into product development. In: Proceedings of the 18th International Conference on Engineering Design 2011 (ICED 2011), Kopenhagen, Denmark, August 15-18, vol. 9, pp. 1-10 (2011)

[15] Abramovici, M., Lindner, A., Krause, F.L.: Providing Product Use Knowledge for the Design of Improved Product Generations. CIRP Annals - Manufacturing Technology 60(1), 211-214 (2011)

[16] Abramovici, M., Neubach, M., Fathi, M., Holland, A.: Knowledge-based Feedback of product Use Information into Product Development. In: Proceedings of the 17th International Conference on Engineering Design (ICED), Stanford, CA, USA, vol. 8, pp. 227238 (2009)

[17] Meier, H.: Ganzheitliches, regelbasiertes Verfügbarkeitsmanagement von Produktionssystemen (VeraPro). Apprimus Verlag, Aachen (2009) ISBN: 978-3-940565-99-0

[18] Abramovici, M., Neubach, M., Fathi, M., Holland, A.: Competing Fusion for Bayesian Applications. In: Proceedings of the 12th International Conference on Information Processing and Management of Uncertainty in Knowledge-Based Systems, Málaga, Spain (2008)

[19] Abramovici, M., Michele, J., Neubach, M.: Erweiterung des PLM-Ansatzes Für Hybride Leistungsb ündel. In: ZWF, vol. 103, pp. 619-621. Carl Hanser Verlag (2008)

[20] Wang, S.Z.: Research on Executive Information System (EIS) for Enterprises. Jianton Unversity Xi'an, Doctoral Thesis (1999)

[21] Leidner, D., Elam, J.: Executive Information Systems: Their Impact on Executive Decision Making. Journal of Management Information Systems 10, 139-155 (1994)

[22] Abramovici, M., Jin, F.: A New Method for Monitoring Industrial Product-Service System based on DSC and AHP. In: Proceedings of ICEIS 2011, Beijing, China (2011)

[23] Michael, M., Bellalouna, F., Schulze, M., Spura, C.: Metadata Reference Model for IPS ${ }^{2}$ Lifecycle Management. In: Proceedings of the 1st CIRP IPS ${ }^{2}$ Conference, Cranfield, UK, pp. 268-272 (2009) 